\title{
Macroscopic Findings Reason Not Done
}

National Cancer Institute

\section{Source}

National Cancer Institute. Macroscopic Findings Reason Not Done. NCI Thesaurus. Code C119865.

The explanation given as to why a macroscopic findings assessment was not performed. 